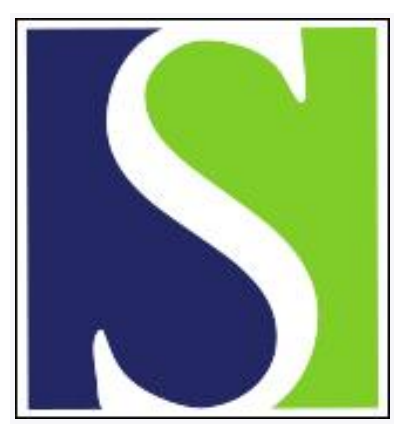

Scand J Work Environ Health 1996;22(1):45-54

https://doi.org/10.5271/sjweh.108

Issue date: Feb 1996

Work conditions and mental health among prison staff in France

by Goldberg P, David S, Landre MF, Goldberg M, Dassa S, Fuhrer R

The following article refers to this text: 2012;38(3):218-227

Key terms: Center for Epidemiologic Studies Depression Scales; epidemiologic study; sleep disorder; social relationship; State Trait Anxiety Inventory

This article in PubMed: www.ncbi.nlm.nih.gov/pubmed/8685673

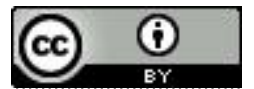




\title{
Work conditions and mental health among prison staff in France
}

\author{
by Paquerette Goldberg, PhD, ${ }^{1}$ Simone David, MS, ${ }^{1}$ Marie F Landre, MS, ${ }^{1}$ Marcel Goldberg, PhD, ${ }^{1}$ \\ Sami Dassa, MS, ${ }^{2}$ Rebecca Fuhrer, PhD ${ }^{3}$
}

\author{
Goldberg P, David S, Landre MF, Goldberg M, Dassa S, Fuhrer R. Work conditions and mental health among \\ prison staff in France. Scand J Work Environ Health 1996;22:45-54.
}

\begin{abstract}
Objectives A cross-sectional epidemiologic survey was conducted among prison staff in France to investigate the relationships between work conditions and mental health.

Methods The sample included men and women 20 to 64 years of age belonging to all categories of prison personnel (prison guards, administrative staff, socioeducational workers, technicians, health care workers, and managers). A postal self-administered questionnaire was used to assess sociodemographic factors, work conditions, and physical and mental disorders. Multiple logistic regression analyses were conducted to determine the effects of work conditions and social relationships on the mental health of prison staff.

Results The results presented in this report only concern depressive symptomatology (measured by the French version of the Center for Epidemiologic Studies Depression Scale), anxiety (measured by the state version of the State-Trait Anxiety Inventory), and sleep disorders. The percentage of mental disorders was higher among prison staff than that determined for other occupational samples. Guards comprised the prison staff least affected by these symptoms.

Conclusions The results show that, in our sample, the factors concerning the subjective evaluation of work conditions and social support were more closely related to mental disorders than work conditions. In addition, seniority was associated with depressive symptoms and anxiety among the men.
\end{abstract}

Key terms Center for Epidemiologic Studies Depression Scales, epidemiologic study, sleep disorders, social relationships, State Trait Anxiety Inventory.

Work conditions in prisons include various sources of stress and rigorous constraints which are relatively specific to the prison environment (1). They include a closed coercive work environment, the need to deal with violence and perform arduous tasks, an occupation dependent on the maintenance of security and order inside prisons, as well as more general constraints affecting, in particular, the organization of work, such as certain work schedules, and relationships within the prison hierarchy.

Several studies of prison guards have already shown the associations between work conditions and mental health. In Australia, Webster et al (2) showed that sleeping tablet consumption was higher among prison guards than among the general population and that more prison guards were subject to psychological difficulties. In New Zealand, Long et al (3) observed that there was more stress among prison staff than among army personnel, but that among prison staff the degree of stress differed depending on the occupational category. In Great Britain, the study by Rutter \& Fielding showed the parts respectively played by the organization of work (4) and relationships with the inmates (5) in the development of stress among prison guards. A study conducted in Finland (6) also showed that the risk of stress among prison personnel is linked to the organization of work and to prison characteristics such as category and size. Two American studies of prison personnel $(7,8)$ revealed the preponderance of occupational over individual characteristics in causing the job burnout syndrome.

Many problems concerning the associations between mental health and work in prisons have not yet been

1 Institut National de la Santé Et de la Recherche Médicale (INSERM, National Institute for Health and Medical Research) Unité 88, Paris, France.

2 Centre National de la Recherche Scientifique, Mutations Industrielles: Rapports Sociaux Homme et Travail (CNRS MIRSHT, National Institute for Scientific Research, Industrial Mutations: Sociological Relationships Man and Work), Paris, France.

3 INSERM U360, Villejuif, France.

Reprint requests to: Dr P Goldberg, Institut National de la Santé Et de la Recherche Médicale, Unité 88, 14 rue du Val d’Osne, 94410 Saint Maurice, France. 
clarified. Little study has been devoted to the roles of various personal and nonoccupational factors, of work characteristics such as the organization of tasks, physical constraints, and relationships with colleagues, professional experts and inmates, or to the part played by social support both at work and outside the work environment, and the potentially diverse effects of all these factors on the different categories of staff. The occupational and nonoccupational environments provide stressors which can be related to the state of health, as well as stress moderators such as social support, as was shown by Karasek et al in their study of Swedish employees (9).

The objectives of the present study were to analyze the relationships between certain aspects of mental health, such as the symptoms of depression, anxiety, and sleep disorders, and the occupational factors characterizing each category of personnel. The goal was to find broad patterns of association by comparing the magnitude of effects of stressors and stress moderators from both the work and the nonwork environment.

\section{Population and methods}

\section{Type of study}

The survey was cross-sectional and was conducted via a self-administered questionnaire mailed to a random sample population designed to represent all of the occupational categories among prison staff in France.

\section{Sample}

The source population comprised about 17000 members of the French Prison Administration, who were divided into the following six occupational categories: prison guards (11 000 men and 2000 women), administrative staff, socioeducational, technical and health care workers, and management. As male prison guards make up about $65 \%$ of the total population, one out of two male guards was randomly selected for inclusion in the study, whereas in the other categories, the entire group was included. In all, 10039 subjects were contacted in January and February 1991, the response rate was $45.7 \%$, and the number of valid questionnaires was 4587 .

The sample consisted of 3474 men and 1113 women. The mean age was 37.1 (SD 8.6) years for the men and 35.0 (SD 8.3) years for the women. Among the men, prison guards represented the largest occupational category ( $84 \%$ of the men). The largest category among the women was that of administrative personnel $(40 \%)$, whereas $32 \%$ were prison guards.

The largest proportions of the sample population ( $55 \%$ of the men and $45 \%$ of the women) were working in establishments for prisoners awaiting judgment or for those sentenced to terms of less than one year. The type of prison less frequently represented was the central prison designed for those serving long terms.

\section{Data}

In all, the questionnaire included 135 questions concerning the occupational and nonoccupational environments and the subject's experience of specific work conditions. As regards work conditions which were not specific to the prison environment, we included questions already used and validated in a large national health survey on work conditions in France (10); these questions dealt with the work schedule (including night work), work commute, posture, noise, heat, lighting, lifting, dust, exposure to chemicals, and so on. For work conditions particular to prisons, we developed specific questions aimed at identifying work stressors and stress moderators and attempted to take into account what seemed important to the workers.

Depending on the specific question, the following three types of response categories were used: quantitative (ie, "During the last month, how many days have you worked without a day off?"); yes/no (ie, "During the last month, did you perform the following tasks?"); and ordinal, using a six-point scale going from none to most (ie, "Do you feel that your professional relationships with the following type of person are frequent?").

The following two states of mental health were evaluated with standardized scales: depressive symptomatology, which was measured by the CES-D scale (Center for Epidemiologic Studies - Depression Scale) $(11,12)$, and the state of anxiety, measured by the STAI (StateTrait Anxiety Inventory) (13) using the version describing the subject's state as opposed to anxiety as a personality trait.

The CES-D scale consists of 20 questions describing symptoms or behavior connected with depression, and a total score is computed from the responses coded from 0 to 3 . We used the thresholds established for the French population (12), 17 or more for men and 23 or more for women, to identify individuals with an elevated level of depressive symptomatology. The STAI evaluates the state of anxiety and was measured according to a similar principle (13); we defined the threshold at 45 or more as indicative of a high level of anxiety. Variations in this threshold did not modify the results.

The questions concerning sleep disorders were derived from the questionnaire developed by Niedhammer et al and validated in France (14). A score was calculated, and sleep disorders were considered to be present when the score was 2 or more, the threshold being based on the distribution of the scores.

\section{Statistical analysis}

The number of variables generated by the different modalities of response to the questionnaire was reduced 
during the first stage of the analysis, using factorial correspondence analyses (15). The main principles of factorial correspondence analysis are as follows. The main inertia axes of the set of points in the subjects' space are computed, and a section through the planes defined by the main inertia axes gives the distance between questions and thereby suggests the grouping of variables. For instance, the "social support at work" variable used in the subsequent analyses was constructed from seven variables totaling 36 modalities. In that specific example, we found that the best possible grouping of variables was a score; a histogram of the distribution of that score allowed us to define a threshold above which social support at work was satisfactory. For other variables, such as work schedule, the findings of the factorial analysis showed that the best grouping was based on identifying a heavy schedule each time there is a positive answer to any of the six questions used for describing the work schedule. Factorial correspondence analyses made it possible to reduce the number of variables used in the subsequent analyses (correspondence analysis not shown). In addition to the health variables, the data after reduction were grouped into 53 variables corresponding to domains describing the occupational and nonoccupational environments.

The method of statistical analysis used to study the relations between the mental health variables and the environmental factors was a multiple logistic regression model derived from the procedure proposed for large numbers of variables by Hosmer \& Lemeshow (16).
EGRET (17) was used to estimate the parameters, and Wald's test was used to compute the P-values.

Two models were studied. One concerned the nonoccupational environment (ie, the variables grouped into three domains: living conditions, nonoccupational relationships and individual factors) and the other concerned the occupational environment (ie, the variables belonging to four domains: professional history, work conditions, subjective experience of work conditions and occupational relationships).

The models were constructed in three stages. The first stage consisted of selecting the control variables from the individual characteristics of the population studied (ie, age, educational level and residential region), which were considered as potential confounding variables. For this purpose, we applied logistic regression to these variables and retained as control variables those for which the P-value was less than 0.05 . The second stage consisted of selecting the variables in each domain for inclusion in the final model. For this purpose we applied logistic regression separately for each domain and the control variables and only retained the domain variables for which the P-value was less than 0.30 .

The last stage was that of constructing the final model on the basis of the variables selected during the first two stages. For this purpose, we included in the model the variables for which the P-value was less than 0.30 , as well as the control variables.

We then explored the links between the occupational environment variables and the mental health disorders

Table 1. Subjects exhibiting depressive symptomatology, a state of anxiety, or sleep disorders according to various individual characteristics.

\begin{tabular}{|c|c|c|c|c|c|c|c|c|c|c|c|c|c|c|}
\hline \multirow[t]{3}{*}{ Characteristics } & \multicolumn{7}{|c|}{ Men } & \multicolumn{7}{|c|}{ Women } \\
\hline & \multicolumn{2}{|c|}{$\begin{array}{c}\text { Depressive } \\
\text { symptomatology }\end{array}$} & \multicolumn{2}{|c|}{ Anxiety } & \multirow[t]{2}{*}{ Meanc } & \multicolumn{2}{|c|}{$\begin{array}{l}\text { Sleep } \\
\text { disorders }\end{array}$} & \multicolumn{2}{|c|}{$\begin{array}{c}\text { Depressive } \\
\text { symptomatology }\end{array}$} & \multicolumn{2}{|c|}{ Anxiety ${ }^{b}$} & \multirow[t]{2}{*}{ Mean ${ }^{\mathbf{b}}$} & \multicolumn{2}{|c|}{$\begin{array}{c}\text { Sleep } \\
\text { disorders }\end{array}$} \\
\hline & $\mathrm{N}$ & $\%$ & $N$ & $\%$ & & $\mathrm{~N}$ & $\%$ & $\mathrm{~N}$ & $\%$ & $\mathrm{~N}$ & $\%$ & & $\mathrm{~N}$ & $\%$ \\
\hline \multicolumn{15}{|l|}{ Age } \\
\hline $\begin{array}{l}20-30 \text { years } \\
31-40 \text { years } \\
41-64 \text { years } \\
\text { Total }\end{array}$ & $\begin{array}{l}159 \\
334 \\
345 \\
838\end{array}$ & $\begin{array}{l}18.1 \\
25.3 \\
29.6\end{array}$ & $\begin{array}{l}148 \\
301 \\
297 \\
746\end{array}$ & $\begin{array}{l}16.8 \\
22.9 \\
25.9\end{array}$ & $\begin{array}{l}33.9 \\
36.4 \\
36.9 \\
36.0\end{array}$ & $\begin{array}{r}308 \\
557 \\
548 \\
1413\end{array}$ & $\begin{array}{l}34.5 \\
41.2 \\
44.6\end{array}$ & $\begin{array}{r}76 \\
88 \\
65 \\
229\end{array}$ & $\begin{array}{l}20.4 \\
19.6 \\
26.3\end{array}$ & $\begin{array}{r}117 \\
137 \\
83 \\
337\end{array}$ & $\begin{array}{l}31.6 \\
31.3 \\
33.3\end{array}$ & $\begin{array}{l}39.5 \\
39.1 \\
40.2 \\
39.5\end{array}$ & $\begin{array}{l}158 \\
207 \\
140 \\
505\end{array}$ & $\begin{array}{l}41.4 \\
44.2 \\
53.2\end{array}$ \\
\hline \multicolumn{15}{|l|}{ Occupation } \\
\hline $\begin{array}{l}\text { Guards } \\
\text { Administration } \\
\text { Socioeducation } \\
\text { Technicians } \\
\text { Management } \\
\text { Medical }\end{array}$ & $\begin{array}{r}701 \\
35 \\
38 \\
46 \\
14 \\
4\end{array}$ & $\begin{array}{l}24.9 \\
25.5 \\
25.3 \\
26.7 \\
20.3 \\
25.0\end{array}$ & $\begin{array}{r}623 \\
36 \\
30 \\
40 \\
12 \\
5\end{array}$ & $\begin{array}{l}22.2 \\
27.3 \\
20.3 \\
23.4 \\
17.1 \\
35.7\end{array}$ & $\begin{array}{l}35.8 \\
38.2 \\
37.6 \\
36.2 \\
35.5 \\
36.6\end{array}$ & $\begin{array}{r}1242 \\
40 \\
41 \\
66 \\
21 \\
3\end{array}$ & $\begin{array}{l}42.6 \\
28.8 \\
27.2 \\
36.3 \\
30.0 \\
18.8\end{array}$ & $\begin{array}{r}66 \\
107 \\
35 \\
3 \\
2 \\
16\end{array}$ & $\begin{array}{r}19.5 \\
25.1 \\
16.2 \\
33.3 \\
7.4 \\
30.2\end{array}$ & $\begin{array}{r}93 \\
155 \\
57 \\
4 \\
3 \\
25\end{array}$ & $\begin{array}{l}27.6 \\
36.6 \\
27.4 \\
36.4 \\
11.5 \\
48.1\end{array}$ & $\begin{array}{l}38.2 \\
40.9 \\
38.4 \\
41.1 \\
34.7 \\
43.1\end{array}$ & $\begin{array}{r}182 \\
193 \\
89 \\
5 \\
8 \\
28\end{array}$ & $\begin{array}{l}51.6 \\
43.5 \\
39.9 \\
45.5 \\
29.6 \\
50.9\end{array}$ \\
\hline Total & 838 & & 746 & & 36.0 & 1413 & & 229 & & 337 & & 39.5 & 505 & \\
\hline \multicolumn{15}{|l|}{ Education } \\
\hline $\begin{array}{l}\text { Earned certificate }{ }^{d} \\
\text { Did not earn certificate }\end{array}$ & $\begin{array}{l}182 \\
655\end{array}$ & $\begin{array}{l}24.1 \\
25.2\end{array}$ & $\begin{array}{l}162 \\
584\end{array}$ & $\begin{array}{l}21.7 \\
22.6\end{array}$ & $\begin{array}{l}36.2 \\
35.9\end{array}$ & $\begin{array}{r}243 \\
1167\end{array}$ & $\begin{array}{l}31.8 \\
43.2\end{array}$ & $\begin{array}{r}134 \\
95\end{array}$ & $\begin{array}{l}20.7 \\
22.7\end{array}$ & $\begin{array}{l}204 \\
133\end{array}$ & $\begin{array}{l}32.1 \\
31.7\end{array}$ & $\begin{array}{l}39.7 \\
39.3\end{array}$ & $\begin{array}{l}292 \\
211\end{array}$ & $\begin{array}{l}43.7 \\
47.7\end{array}$ \\
\hline Total & 837 & & 746 & & 36.0 & 1410 & & 229 & & 337 & & 39.5 & 503 & \\
\hline
\end{tabular}

a Threshold: score $\geq 17$ for the men, $\geq 23$ for the women.

b Threshold: score $\geq 45$.

- Mean score of anxiety scale.

d Baccalaureat or French School Leaving Certificate. 
Table 2. Models for depressive symptomatology - results of logistic regression analyses. ${ }^{a}(\mathrm{OR}=$ odds ratio, $95 \% \mathrm{Cl}=95 \%$ confidence interval)

\begin{tabular}{|c|c|c|c|}
\hline Model & P-value & OR & $95 \% \mathrm{Cl}$ \\
\hline \multicolumn{4}{|l|}{ Occupational model } \\
\hline \multicolumn{4}{|l|}{$M^{b}{ }^{b}$} \\
\hline \multicolumn{4}{|l|}{ Prior experience } \\
\hline No & & 1 & \\
\hline Yes & 0.007 & 0.67 & $0.50-0.89$ \\
\hline \multicolumn{4}{|l|}{ Seniority } \\
\hline $0-4$ years & & 1 & \\
\hline $5-9$ years & 0.66 & 0.93 & $0.67-1.29$ \\
\hline $10-14$ years & 0.046 & 1.45 & $1.01-2.08$ \\
\hline$\geq 15$ years & $<0.001$ & 2.17 & $1.47-3.22$ \\
\hline \multicolumn{4}{|l|}{ Type of prison } \\
\hline Long-term prisoners & & 1 & \\
\hline Short-term prisoners & 0.21 & 1.14 & $0.93-1.40$ \\
\hline Administration & 0.01 & 1.65 & $1.12-2.44$ \\
\hline \multicolumn{4}{|l|}{ Tasks alternating } \\
\hline Satisfactory & & & \\
\hline Unsatisfactory & $<0.001$ & 1.58 & $1.31-1.90$ \\
\hline \multicolumn{4}{|c|}{ Problems with inmate behavior } \\
\hline Yes & & & \\
\hline No & 0.04 & 1.25 & $1.01-1.55$ \\
\hline \multicolumn{4}{|l|}{ Difficult schedules } \\
\hline No & & 1 & \\
\hline Yes irregular & 0.19 & 1.17 & $0.93-1.46$ \\
\hline \multirow{2}{*}{\multicolumn{4}{|c|}{ Work satisfaction }} \\
\hline & & & \\
\hline $\begin{array}{l}\text { Yes } \\
\text { No }\end{array}$ & $<0.001$ & 1.64 & $1.35-2.00$ \\
\hline \multicolumn{4}{|l|}{ Professional image } \\
\hline Positive & & 1 & \\
\hline Negative & $<0.001$ & 1.59 & $1.25-2.03$ \\
\hline \multicolumn{4}{|c|}{ Quality frequency relationships } \\
\hline No & & 1 & \\
\hline Yes & $<0.001$ & 0.68 & $0.57-0.81$ \\
\hline \multicolumn{4}{|l|}{ Support at work } \\
\hline Unsatisfactory & & 1 & \\
\hline Satisfactory & $<0.001$ & 0.59 & $0.46-0.76$ \\
\hline \multicolumn{4}{|l|}{ Womenc } \\
\hline \multicolumn{4}{|l|}{ Expression of difficulties } \\
\hline No & & 1 & \\
\hline Yes & 0.02 & 1.47 & $1.06-2.04$ \\
\hline \multicolumn{4}{|l|}{ Work satisfaction } \\
\hline Yes & & 1 & \\
\hline No & 0.01 & 1.60 & $1.11-2.29$ \\
\hline Consideration at work & & & \\
\hline No & & 1 & \\
\hline Yes & 0.04 & 0.64 & $0.42-0.99$ \\
\hline Support at work & & & \\
\hline Unsatisfactory & & 1 & \\
\hline Satisfactory & 0.001 & 0.47 & $0.30-0.74$ \\
\hline Nonoccupational model & & & \\
\hline Men & & & \\
\hline $\begin{array}{l}\text { Occupational category } \\
\text { Guard }\end{array}$ & & & \\
\hline Administrative & 0.08 & 1.50 & $0.95-2.35$ \\
\hline Socioeducator & 0.03 & 1.60 & $1.04-2.46$ \\
\hline Technician & 0.16 & 1.33 & $0.89-1.97$ \\
\hline Manager & 0.36 & 1.34 & $0.71-2.52$ \\
\hline Medical & 0.92 & 1.06 & $0.31-3.68$ \\
\hline Women & & & \\
\hline Occupational category & & & \\
\hline Guard & & & \\
\hline Administrative & 0.001 & 2.02 & $1.35-3.04$ \\
\hline $\begin{array}{l}\text { Socioeducator } \\
\text { Technician }\end{array}$ & $\begin{array}{l}0.20 \\
0.12\end{array}$ & $\begin{array}{l}1.42 \\
3.62\end{array}$ & $\begin{array}{l}0.83-2.40 \\
0.72-18.1\end{array}$ \\
\hline Manager & 0.43 & 0.55 & $0.12-2.45$ \\
\hline Medical & 0.03 & 2.28 & $1.10-4.74$ \\
\hline
\end{tabular}

a Only significant variables are shown $(P \leq 0.05)$.

b Adjustment for age.

c No adjustment variable. under study to see if they were modified, and therefore partly explained, by the significant variables of the nonoccupational model. For this purpose, the significant nonoccupational variables were introduced one by one into the occupational model, and the resulting changes in the odds ratios (OR) were examined.

Last, as within each occupational category, the distribution of work situations and conditions were fairly homogeneous, we calculated the odds ratios for each occupational category by introducing this variable into the nonoccupational model. By means of its odds ratio, this variable, which was linked in a complex fashion to the occupational variables, gave an overall view of the situation of each occupational category in relation to the health disorders studied.

We performed separate analyses for the men and the women because there could be gender differences between either the prisons or the guards. Indeed, within the prison guard category, the job contents varied according to the type of prison (for those serving long terms and for those awaiting judgment or sentenced to terms of less than one year), and female prison guards work almost only in prisons for women.

\section{Results}

\section{Depressive symptomatology}

Complete data were available for $97 \%$ of the sample. The overall proportion of subjects with depressive symptomatology was $24 \%$ (men $24.9 \%$, women $21.4 \%$ ).

Table 1 shows the percentage of persons exhibiting depressive symptomatology, according to the main characteristics of the sample. Age played a part in these symptoms for both genders, but especially for the men. Guards did not report more depressive symptomatology than the other categories. The management staff reported the lowest frequency of this disorder. The categories most affected seemed to be the women among the health care workers and technicians $(30.2 \%$ and $33.3 \%$, respectively). There was little difference according to educational level.

Table 2 gives the results for the fitted occupational model. For the men the odds ratios were adjusted for age, as the other variables initially considered for adjustment were not significant at the $5 \%$ level. No adjustment was made for the women. Results are only given for the significant variables $(\mathrm{P} \leq 0.05)$.

For the men, the occupational model shows the increased effect of seniority in the prison administration, with a clear gradient. Work conditions (type of prison, unsatisfactory alternating task, and problems with inmate behavior) and subjective experience with work con- 
Table 3. Models for state of anxiety - results of logistic regression analyses. ${ }^{a}(O R=$ odds ratio, $95 \% \mathrm{Cl}=95 \%$ confidence interval)

\begin{tabular}{|c|c|c|c|}
\hline Model & P-value & $\mathrm{OR}$ & $95 \% \mathrm{Cl}$ \\
\hline \multicolumn{4}{|l|}{ Occupational model } \\
\hline \multicolumn{4}{|l|}{$\operatorname{Men}^{\mathrm{b}}$} \\
\hline \multicolumn{4}{|l|}{ Unemployment } \\
\hline $\begin{array}{l}\text { No } \\
\text { Yes }\end{array}$ & 0.001 & $\begin{array}{l}1 \\
1.42\end{array}$ & $1.14-1.77$ \\
\hline \multicolumn{4}{|l|}{ Seniority } \\
\hline $\begin{array}{l}0-4 \text { years } \\
5-9 \text { years } \\
10-14 \text { years } \\
\geq 15 \text { years }\end{array}$ & $\begin{aligned} & 0.43 \\
& 0.009 \\
< & 0.001\end{aligned}$ & $\begin{array}{l}1 \\
1.15 \\
1.65 \\
2.06\end{array}$ & $\begin{array}{l}0.82-1.60 \\
1.14-2.40 \\
1.37-3.08\end{array}$ \\
\hline \multicolumn{4}{|l|}{ Task } \\
\hline $\begin{array}{l}\text { Socioeducational } \\
\text { Others } \\
\text { Detention }\end{array}$ & $\begin{array}{l}0.17 \\
0.12\end{array}$ & $\begin{array}{l}1 \\
1.24 \\
0.80\end{array}$ & $\begin{array}{l}0.91-1.69 \\
0.61-1.06\end{array}$ \\
\hline \multicolumn{4}{|l|}{ Tasks alternating } \\
\hline $\begin{array}{l}\text { Satisfactory } \\
\text { Unsatisfactory }\end{array}$ & $<0.001$ & $\begin{array}{l}1 \\
1.47\end{array}$ & $1.21-1.78$ \\
\hline \multicolumn{4}{|c|}{ Commute home-work site } \\
\hline $\begin{array}{l}\text { Normal } \\
\text { Long }\end{array}$ & $<0.01$ & $\begin{array}{l}1 \\
1.38\end{array}$ & $1.14-1.66$ \\
\hline \multicolumn{4}{|l|}{ Difficult schedules } \\
\hline $\begin{array}{l}\text { No } \\
\text { Yes irregular } \\
\text { Yes too long }\end{array}$ & $\begin{array}{l}0.03 \\
0.004\end{array}$ & $\begin{array}{l}1 \\
1.32 \\
1.50\end{array}$ & $\begin{array}{l}1.03-1.70 \\
1.14-1.99\end{array}$ \\
\hline \multicolumn{4}{|c|}{ Interest and responsibility } \\
\hline $\begin{array}{l}\text { Great } \\
\text { Small }\end{array}$ & 0.02 & $\begin{array}{l}1 \\
1.64\end{array}$ & $1.08-2.50$ \\
\hline \multicolumn{4}{|l|}{ Work satisfaction } \\
\hline $\begin{array}{l}\text { Yes } \\
\text { No }\end{array}$ & $<0.001$ & $\begin{array}{l}1 \\
1.81\end{array}$ & $1.48-2.21$ \\
\hline \multicolumn{4}{|l|}{ Professional image } \\
\hline $\begin{array}{l}\text { Positive } \\
\text { Negative }\end{array}$ & $<0.001$ & $\begin{array}{l}1 \\
1.55\end{array}$ & $1.21-2.00$ \\
\hline \multicolumn{4}{|c|}{ Quality frequency relationships } \\
\hline $\begin{array}{l}\text { No } \\
\text { Yes }\end{array}$ & 0.006 & $\begin{array}{l}1 \\
0.77\end{array}$ & $0.64-0.92$ \\
\hline \multicolumn{4}{|l|}{ Dependency at work } \\
\hline $\begin{array}{l}\text { Yes } \\
\text { No }\end{array}$ & 0.047 & $\begin{array}{l}1 \\
0.77\end{array}$ & $0.60-0.99$ \\
\hline \multicolumn{4}{|l|}{ Support at work } \\
\hline $\begin{array}{l}\text { Unsatisfactory } \\
\text { Satisfactory }\end{array}$ & $<0.001$ & $\begin{array}{l}1 \\
0.52\end{array}$ & $0.41-0.67$ \\
\hline \multicolumn{4}{|l|}{ Womenc } \\
\hline \multicolumn{4}{|l|}{ Satisfactory hygiene } \\
\hline $\begin{array}{l}\text { Yes } \\
\text { No }\end{array}$ & 0.02 & $\begin{array}{l}1 \\
1.46\end{array}$ & $1.05-2.02$ \\
\hline \multicolumn{4}{|l|}{ Difficult situations } \\
\hline $\begin{array}{l}\text { No } \\
\text { Yes } \\
\text { No response }\end{array}$ & $\begin{array}{l}0.10 \\
0.99\end{array}$ & $\begin{array}{l}1 \\
0.62 \\
1.01\end{array}$ & $\begin{array}{l}0.35-1.10 \\
0.56-1.79\end{array}$ \\
\hline \multicolumn{4}{|c|}{ Expression of difficulties } \\
\hline $\begin{array}{l}\text { No } \\
\text { Yes }\end{array}$ & 0.008 & $\begin{array}{l}1 \\
1.48\end{array}$ & $1.11-1.97$ \\
\hline \multicolumn{4}{|l|}{ Work satisfaction } \\
\hline $\begin{array}{l}\text { Yes } \\
\text { No }\end{array}$ & 0.03 & $\begin{array}{l}1 \\
1.45\end{array}$ & $1.04-2.03$ \\
\hline \multicolumn{4}{|l|}{ Consideration at work } \\
\hline $\begin{array}{l}\text { No } \\
\text { Yes }\end{array}$ & 0.04 & $\begin{array}{l}1 \\
0.65\end{array}$ & $\begin{array}{l}0.44-0.97 \\
\text { (continued }\end{array}$ \\
\hline
\end{tabular}

Table 3. Continued

\begin{tabular}{llll}
\hline Model & P-value & OR & $95 \% \mathrm{Cl}$ \\
\hline Nonoccupational model & & & \\
Men & & & \\
Occupational category & & & \\
$\quad$ Guard & & 1 & \\
$\quad$ Administrative & 0.003 & 1.99 & $1.27-3.12$ \\
$\quad$ Socioeducator & 0.16 & 1.39 & $0.88-2.20$ \\
$\quad$ Technician & 0.34 & 1.28 & $0.85-1.92$ \\
$\quad$ Manager & 0.53 & 1.24 & $0.64-2.41$ \\
$\quad$ Medical & 0.15 & 2.38 & $0.73-7.76$ \\
Women & & & \\
Occupational category & & & \\
$\quad$ Guard & & 1 & \\
$\quad$ Administrative & 0.003 & 1.62 & $1.18-2.23$ \\
$\quad$ Socioeducator & 0.59 & 1.12 & $0.75-1.66$ \\
$\quad$ Technician & 0.40 & 1.73 & $0.48-6.24$ \\
$\quad$ Manager & 0.16 & 0.41 & $0.11-1.43$ \\
$\quad$ Medical & 0.01 & 2.23 & $1.21-4.11$ \\
\hline
\end{tabular}

a Only significant variables are shown $(P \leq 0.05)$.

b Adjustment for age.

- No adjustment variable.

ditions (difficult schedules, poor work satisfaction and negative professional image) were associated with depressive symptomatology, as well as with occupational relationships, which acted as protective factors, with odds ratios below one for the quality and frequency of the relationships and support at work.

For the women, the variables showing an association with depressive symptomatology reflected subjective experience with work conditions (expression of difficulties and poor satisfaction at work), whereas consideration and support at work played a protective role.

For both the men and the women, the introduction of significant nonoccupational factors into the occupational model only led to slight modifications (analysis not shown).

Finally, the odds ratios concerning the occupational category confirmed that fewer prison guards than other staff reported depressive symptomatology when the nonoccupational environment was taken into account. Among the men, this disorder seemed to characterize the socioeducational and administrative staff, and, among the women, it described the medical and administrative staff, the values of the odds ratios being higher than among the men.

\section{Anxiety}

Complete data were available for $96 \%$ of the sample. The percentage of the subjects exhibiting a state of anxiety was $24.6(22.3 \%$ for the men and $31.9 \%$ for the women). The general mean STAI score was 36.8 ( 36 for the men and 39.5 for the women).

For the men only, there was a positive association between age and anxiety (table 1). As for depressive symptomatology, the prison guard category was not more 
affected by anxiety than the other categories. Among the men, the most affected were the administrative and the socioeducational staff. Among the women, administrative personnel also reported anxiety, but the most affected were the health care staff. As in the case of depressive symptomatology, educational level did not appear to be related to report of anxiety.

Table 4. Models for sleep disorders - results of logistic regression analyses. ${ }^{\text {a }}(\mathrm{OR}=$ odds ratio, $95 \% \mathrm{Cl}=95 \%$ confidence interval)

\begin{tabular}{|c|c|c|c|}
\hline Model & P-value & OR & $95 \% \mathrm{Cl}$ \\
\hline \multicolumn{4}{|l|}{ Occupational model } \\
\hline \multicolumn{4}{|l|}{$\operatorname{Men}^{b}$} \\
\hline \multicolumn{4}{|l|}{ Alternating tasks } \\
\hline $\begin{array}{l}\text { Satisfactory } \\
\text { Unsatisfactory }\end{array}$ & $<0.001$ & $\begin{array}{l}1 \\
1.35\end{array}$ & $1.14-1.59$ \\
\hline \multicolumn{4}{|l|}{ Constraints } \\
\hline $\begin{array}{l}\text { No } \\
\text { Yes }\end{array}$ & $<0.001$ & $\begin{array}{l}1 \\
1.33\end{array}$ & $1.13-1.56$ \\
\hline \multicolumn{4}{|l|}{ Difficult schedules } \\
\hline $\begin{array}{l}\text { No } \\
\text { Yes irregular } \\
\text { Yes too long }\end{array}$ & $\begin{array}{l}0.01 \\
0.009\end{array}$ & $\begin{array}{l}1 \\
1.29 \\
1.36\end{array}$ & $\begin{array}{l}1.06-1.56 \\
1.08-1.70\end{array}$ \\
\hline \multicolumn{4}{|l|}{ Work satisfaction } \\
\hline $\begin{array}{l}\text { Yes } \\
\text { No }\end{array}$ & 0.03 & $\begin{array}{l}1 \\
1.21\end{array}$ & $1.02-1.43$ \\
\hline \multicolumn{4}{|l|}{ Professional image } \\
\hline $\begin{array}{l}\text { Positive } \\
\text { Negative }\end{array}$ & $<0.001$ & $\begin{array}{l}1 \\
1.54\end{array}$ & $1.28-1.86$ \\
\hline \multicolumn{4}{|l|}{ Support at work } \\
\hline $\begin{array}{l}\text { Satisfactory } \\
\text { Unsatisfactory }\end{array}$ & $<0.001$ & $\begin{array}{l}1 \\
0.62\end{array}$ & $0.49-0.79$ \\
\hline \multicolumn{4}{|l|}{ Women $^{c}$} \\
\hline \multicolumn{4}{|l|}{ Constraints } \\
\hline $\begin{array}{l}\text { No } \\
\text { Yes }\end{array}$ & 0.03 & $\begin{array}{l}1 \\
1.37\end{array}$ & $1.02-1.83$ \\
\hline \multicolumn{4}{|l|}{ Difficult schedules } \\
\hline $\begin{array}{l}\text { No } \\
\text { Yes irregular } \\
\text { Yes too long }\end{array}$ & $\begin{array}{l}0.04 \\
0.04\end{array}$ & $\begin{array}{l}1 \\
1.45 \\
1.41\end{array}$ & $\begin{array}{l}1.02-2.04 \\
1.02-1.94\end{array}$ \\
\hline \multicolumn{4}{|c|}{ Nonoccupational model } \\
\hline \multicolumn{4}{|c|}{ Men } \\
\hline \multicolumn{4}{|c|}{ Occupational category } \\
\hline $\begin{array}{l}\text { Guard } \\
\text { Administrative } \\
\text { Socioeducator } \\
\text { Technician } \\
\text { Manager } \\
\text { Medical }\end{array}$ & $\begin{array}{l}0.26 \\
0.37 \\
0.64 \\
0.52 \\
0.07\end{array}$ & $\begin{array}{l}1 \\
0.79 \\
0.82 \\
0.92 \\
0.83 \\
0.30\end{array}$ & $\begin{array}{l}0.52-1.20 \\
0.53-1.26 \\
0.65-1.30 \\
0.47-1.46 \\
0.08-1.10\end{array}$ \\
\hline \multicolumn{4}{|c|}{ Women } \\
\hline \multicolumn{4}{|c|}{ Occupational category } \\
\hline $\begin{array}{l}\text { Guard } \\
\text { Administrative } \\
\text { Socioeducator } \\
\text { Technician } \\
\text { Manager } \\
\text { Medical }\end{array}$ & $\begin{array}{l}0.70 \\
0.33 \\
0.65 \\
0.27 \\
0.97\end{array}$ & $\begin{array}{l}1 \\
0.94 \\
0.83 \\
0.75 \\
0.61 \\
1.01\end{array}$ & $\begin{array}{l}0.68-1.29 \\
0.56-1.22 \\
0.21-2.60 \\
0.25-1.47 \\
0.56-1.84\end{array}$ \\
\hline
\end{tabular}

- Only significant variables are shown ( $P \leq 0.05)$.

v Adjustment for age and educational level.

- Adjustment for age.
Table 3 presents the results of the occupational model for anxiety. The adjustment variables were the same as for depressive symptomatology.

Among the men, there was a negative effect for seniority in the prison administration, with a gradient. Unemployment was an experience connected with the development of anxiety. The results for the three domains comprising work conditions, subjective experience with work conditions, and relationships at work were very close to those observed for depressive symptomatology. In addition, a long commute between home and work, lack of interest and responsibility, and dependency at work were aggravating factors.

As for depressive symptomatology, fewer occupational variables were associated with a state of anxiety among the women than among the men.

As was the case for depressive symptomatology, the introduction of nonoccupational factors into the occupational model only produced slight changes for the variables of borderline significance (analysis not shown).

Last, the odds ratios for the occupational categories confirmed that, as for depressive symptomatology, fewer prison guards than other staff displayed anxiety when the nonoccupational environment was taken into account. Anxiety seemed, above all, to characterize the health care workers and the administrative staff.

\section{Sleep}

The percentage of the sample with sleep disorders was $41.8 \%$. Fewer men than women stated they had such disorders (40.7 versus $45.4 \%$ ).

The descriptive results (table 1) showed that for both genders, sleep disorders increased with age. For both genders, fewer of those whose educational level had enabled them to obtain at least the Baccalaureate or French School Leaving Certificate (12 years of schooling) had sleep disorders than the others. For both genders, the prison guards formed the category most affected by sleep disorders, and female health care workers were next.

For sleep disorders, the adjustment variables for the occupational logistic regression model were age, and, for the men only, educational level, which showed the protective role of a higher level of education.

In the occupational model (table 4), no factor from the domain representing professional history was included; this result indicated the absence of a link between this historical aspect of occupational life and sleep disorders. Of the work conditions, for the men, those which played a role in sleep pathology concerned the alternation of tasks and the existence of constraints or harmful factors at work. The factors belonging to the domain characterizing subjective experience with work conditions in relation to sleep disorders were the problems of difficult schedules, job dissatisfaction, and a 
negative image of the profession. Relationships at work only intervened via social support and thus showed its protective effect. Among the women, the only variables associated with sleep disorders were job constraints and difficult schedules.

The introduction of significant nonoccupational variables into the occupational model did not modify the latter model in any way (analysis not shown).

As regards occupational categories, the odds ratios confirmed that, for an identical occupational environment, more prison guards, male or female, than members of other categories stated that they had sleep disorders, but the differences between the categories were not significant.

\section{Discussion}

This study of prison staff in France showed a high prevalence of mental health problems among this population. It found that factors concerning subjective experience with work conditions and social relationships at work were closely associated with mental health disturbances.

The voluntary nature of subject participation and the $45 \%$ response rate may, however, have led to a selection bias. For instance, one may wonder whether a larger proportion of those most affected by mental disturbances replied to the questionnaire. Given the completely anonymous nature of the replies - which was very important to maximize the level of participation - it is extremely difficult to give a simple answer to the question of a possible selection bias. In any case, however, the overall rate of participation (45\%) was close to that usually found in France for epidemiologic surveys conducted by mail; thus, for a comparable study of workers in the French electrical industry, the response rate was $47 \%$ (18).

Another bias could be due to the fact that people suffering from mental disturbances may have a more negative judgment of their work conditions and social relationships. However, if such an effect had occurred, one would expect that mental disturbances would have been associated with any judgmental variable, which was not the case.

For depressive symptomatology, the present results were compared with those obtained in France for two groups of electrical industry workers: men whose work involved traveling (19) and a group of men and women, comparable with those in our sample, working in a nuclear power station (20). For these two groups, 14.9 and $14 \%$ of the samples, respectively, exhibited depressive symptomatology, whereas in our sample this disturbance affected $24.9 \%$ of the men and $21.4 \%$ of the women.
The mean score for anxiety, evaluated according to the State-Trait Anxiety Inventory (STAI), for the group of nuclear power station workers was 35 (20), and it was similar for different categories of employees ( 34.3 and 35.7). For our sample, this score was 36 for the men and 39.5 for the women. In another survey using the same instrument and concerning male policemen, firemen and municipal workers, Pendelton et al (21) found mean scores of $34.1,30.7$ and 35.9 , respectively, for anxiety state.

Niedhammer et al (22) published results on sleep disturbance using the same questionnaire for female nurses and aides working in hospitals. These results are comparable with those for the women in our sample, although our sample was a little younger than the women in the Niedhammer survey $(20 \%$ were under 30 years of age in their study compared with $30 \%$ in ours). Nevertheless, more of the women in our sample stated that they had sleep disturbances in their replies to the questions concerning sleep that were comparable in the two surveys. Thus, in our sample, the percentages with disturbances ranged from 11 (light sleepers on days of rest) to 32 (waking up during sleep on work days) whereas, in the hospital nurse group, the corresponding figures ranged from $3 \%$ (difficulty in falling asleep on days of rest) to $17 \%$ (waking up during sleep on workdays).

The relationships between depressive symptomatology, measured by the Center for Epidemiologic Studies Depression Scale (CES-D), and various sociodemographic characteristics have already been studied, and some of the associations established were found among prison staff in this study. Thus three American surveys of the general population $(23-25)$ showed the effects of marital status and income on the development of depressive symptomatology, and this effect was also found in our survey in the nonoccupational models (results not shown). In a survey of Japanese tax administration workers, Iwata et al (26) stressed the negative effects of unsatisfactory social relations and stress, both outside work and in the work environment, on depressive symptomatology. Neither the Japanese survey nor the present study produced the same results as the American surveys for the role played by age and gender in the development of depressive symptomatology. Among the prison staff surveyed in our study, we did not find that a high level of education had an effect on symptomatology, unlike that observed in the American studies.

Two studies of occupational stress among police personnel, by Brown \& Campbell in England (27) and Evans \& Coman in Australia (28), used the STAI as one of the measures of stress. The first used the same version as the one we applied, reflecting the state, and the second used the version reflecting the subject's trait. In both surveys, the authors reported that the development of stress was linked to the organization of work and social relations at 
work, rather than to the nature of the work itself, except for the officers in the English study, for whom the nature of work was also related to stress.

We did not find large differences between the men and women. However, there were fewer significant associations with variables related to work conditions among the women, both for depressive symptomatology and anxiety, and most of the associations were related to the subjective evaluation and to social relationships at work. In addition, there were proportionally more significant associations in the nonoccupational models than in the occupational ones (analyses not shown), implying that the nonwork environment may have a greater importance for mental health among women.

One of the striking results of the present study concerned the fact that prison guards were the least affected by depressive symptomatology and by anxiety. At first, this finding might seem paradoxical, because of the assumption that the prison guards are those most exposed to the different sources of stress and occupational difficulties. It may be partly due to the selection of the staff taken during the most recent period and also to better adaptation to the specific constraints of the prison environment; thus these constraints might be perceived by prison guards as an integral part of their work, whereas other categories might consider them less essential and therefore feel more hampered by them. Our observation of fewer mental health disorders among prison guards, and, among men, those with less seniority in the prison administration, might also be explained by the fact that mental health disorders are not directly related to tasks and work conditions (ie, exposure to inmates' violence and threats) and maintenance of order in prison, but rather by social relationships at work (ie, frequency and quality of relationships and communication with colleagues, superiors and the central administration). This possibility is strengthened by the absence, in our study, of strong effects on depressive symptomatology and anxiety attributable to the variables concerning the nature of the tasks, whereas the effects of the variables reflecting the subjective experience of work conditions and social relations at work were clearly important in this respect, as in the aforementioned English and Australian studies $(27,28)$. It is important to note that, in the present sample, the prison guards constituted the great majority of the men and that consequently the other categories were of marginal significance, both as regards occupational values and social support at work in a prison environment. However, although our study showed that prison guards reported fewer depressive symptoms and anxiety states than other occupational categories, this relationship seemed to weaken with seniority for the men, since, for subjects of the same age, the risk of such symptomatology and anxiety increased evenly with seniority (tables 2 and 3). This result was not due to any change in task content or to the greater responsibilities of the managerial staff, since promotions occurred in the course of the subjects' careers. Thus the risks relating to seniority were almost unchanged when grade was taken into account and, rather, tended towards a slight decrease (results not shown). This decrease can be interpreted as a weakening of the various protective mechanisms, which gradually became less effective with time. The fact that a similar effect, often mentioned in connection with occupational psychopathology (29), was not seen among the women may be due to the lack of statistical power, the number of female guards being small in the sample.

A similar result was found for men in a large study of Swedish prison staff $(30,31)$, in which the level of plasma cortisol was used as an indicator of psychological strain at work. For the men, there was no significant difference between the occupational groups, whereas female guards had the highest mean level of plasma cortisol and they reported more psychiatric symptoms.

The hypothesis given by Cheek \& Miller (32) to explain that the prison guards working in New Jersey (United States) seemed less affected by stress than other occupational groups could also be evoked: the denial of stress leads to minimized reporting of stress-related problems. However the American study found a different result for prevalence ratios of hypertension and ulcers, which were higher for prison guards of New Jersey than for other occupational groups. This was not the case in our sample, after control for the other variables (results not shown).

Some studies of sleep disorders among working and general populations, such as the surveys of workers in small and medium-size businesses in the Paris area (33), of a general working population (34), and of the general French population (35), have shown that gender and age affect self-reported sleep disturbances. Jacquinet-Salord et al (33) and Butat et al (34) studied the effects of certain factors in the work environment on sleep. The Butat study showed the role played by noise, a result found indirectly in our study in the synthesized variable entitled "constraints" at work. In the Jacquinet-Salord study, no relationship was found between work conditions (assembly-line work, work schedules and noise) and sleep pathology. But the results indicated a link between the latter and experience with work conditions, and the same link was found in our study. Thus subjects who reported intellectual and psychological burdens and lack of interest and satisfaction at work stated more sleep disturbance.

Despite the usual limitations of cross-sectional studies conducted with the aid of a questionnaire, our survey corroborated some of the results already reported and enabled us to define the effects of various occupational and nonoccupational factors on the mental health of 
prison staff. The factors connected with experience with work conditions and with social relations, which seem to have a potentially important protective role, should help to orientate the future policies of those responsible for prison management towards integrating more of these factors into the organization of tasks and social relations at work in the prison environment.

\section{Acknowledgments}

This survey was conducted with the help of the Adviser for Research of the French Ministry of Justice and the support of the Management of Labour Relations at the Ministry of Labour.

The authors wish to thank the staff of the French prison administration, as well as the CESDIP (Centre de Recherches Sociologiques sur le Droit et les Institutions Pénales, Sociological Research Center on Law and Prison Institutions) and the Friendly Society of the Ministry of Justice, for their valuable assistance in carrying out this survey.

\section{References}

1. Chauvenet A, Benguigui G, Orlic F. Les surveillants de prison: le prix de la sécurité. R Fr Sociol 1993:34:345-66.

2. Webster IW, Porritt DW, Brennan PI. Reported health, lifestyle and occupational stress in prison officers. Community Health Studies 1983;7(3):266-77.

3. Long N, Shouksmith G, Voges K, Roache S. Stress in prison staff: an occupational study. Criminology 1986;24(2):33145.

4. Rutter DR, Fielding PJ. Sources of occupational stress: an examination of British prison officers. Work Stress 1988;2(4): 291-99.

5. Launay G, Fielding PJ. Stress among prison officers: some empirical evidence based on self report. Howard J 1989;28(2): $138-47$.

6. Kalimo R. Stress in work: conceptual analysis and a study on prison personnel. Scand J Work Environ Health 1980;6 suppl 3.

7. Gerstein LH, Topp CG, Correll G. The role of the environment and person when predicting burnout among correctional personnel. Crim Justice Behav 1987;14(3):352-69.

8. Whitehead JT, Linquist CA. Correctional officer job burnout: a path model. J Res Crime Delinquency 1986;23(1);23- 42.

9. Karasek R, Gardell B, Lindell J. Work and non-work correlates of illness and behaviour in male and female swedish white collar workers. J Occup Behav 1987;8:187-207.

10. Bue J, Cristofari MF. Contraintes et nuisances dans la vie de travail: deuxième série de résultats de l'enquête nationale sur les conditions de travail réalisée en mars 1984. 1986;20:595.

11. Radloff LS. The CES-D scale: a self-report depression scale for research in the general population. Appl Psychol Meas $1977 ; 3: 385-401$.
12. Fuhrer R, Rouillon F. La version française de l'échelle CESD: description et traduction de l'échelle d'autoévaluation [Center for Epidemiologic Studies: depression scale]. Psychiatr Psychobiol 1989;4:163-6.

13. Spielberger CD, Gorsuch RL, Lushene RE. STAI manual for state-trait anxiety inventory. Palo Alto (CA): Consulting Psychologists Press, 1970

14. Niedhammer I, Lert F, Marne MJ. Analyse longitudinale des effets du travail posté sur le sommeil des infirmières. In: International Commission on Occupational Health ( $\mathrm{ICOH})$. 24e Congrès International Santé au Travail, sept-oct 1993. Nice (France): ICOH, 1993:345.

15. Greenacre MJ. Theory and applications of correspondence analysis. London: Academic Press, 1984.

16. Hosmer DW, Lemeshow S. Applied logistic regression. New York (NY): John Wiley and Sons, 1989.

17. Statistics and Epidemiology Research Corporation. EGRET: reference manual. Seattle (WA): Statistics and Epidemiology Research Corporation, 1985.

18. Goldberg M, Leclerc A, Chastang JF, Morcet JF, Marne MJ, Luce $\mathrm{D}$, et al. Mise en place d'une cohorte épidémiologique à Electricité de France-Gaz de France: recrutement des volontaires: principales caractéristiques de l'échantillon: Resp-Informations. Rev Epidemiol Santé Publique 1990;38:265—68, $378-80$.

19. Levene F, Imbernon E, Warret G, Blanc MG, Delpy B, Desorby $\mathrm{P}$, et al. Déplacements professionnels en véhicules EDFGDF et répercussions éventuelles sur l'état de santé. In: International Commission on Occupational Health (ICOH). 24e Congrès International Santé au Travail, sept-oct 1993. Nice (France): ICOH, 1993:110.

20. Telle MA, Huez D, Niedbala JM, Auclair J, Charpak Y, et al. Santé mentale et arrêt de tranche en centrale nucléaire: une étude épidémiologique. Radioprotection. In press.

21. Pendleton M, Scotland E, Spiers P, Kirsch E. Stress and strain among police, firefighters, and government workers: a comparative analysis. Crim Justice Behav 1989;16,2:196--210.

22. Niedhammer I, Lert F, Marne MJ. Effect of driftwork on sleep among French nurses: a longitudinal study. J Occup Med. In press.

23. Comstock GW, Helsing KJ. Symptoms of depression in two communities. Psychol Med 1976;6:551—63.

24. Eaton WW, Kessler LG. Rates of symptoms of depression in a national sample. Am J Epidemiol 1981;114(4):528-38.

25. Frerichs RR, Aneshensel CS, Clark VA. Prevalence of depression in Los Angeles county. Am J Epidemiol 1981;113(6): 691-9.

26. Iwata N, Okuyama Y, Kawakami Y, Saito K. Prevalence of depressive symptoms in a Japanese occupational setting: a preliminary study. Am J Public Health 1989;79(11):1486-9.

27. Brown JM, Campbell EA. Sources of occupational stress in the police. Work Stress 1990;4(4):305-18.

28. Evans BJ, Coman GJ. General versus specific measures of occupational stress: an Australian police survey. Stress Med 1993;9:11-20.

29. Dejours C. Travail, usure mentale: essai de psychopathologie du travail. Paris: Bayard ed, 1993.

30. Härenstam A. Prison personnel - working conditions, stress and health: a study of 2000 prison employees in Sweden [dissertation]. Stockholm: Karolinska Institute, 1989.

31. Härenstam $A$, Theorell $T$. Cortisol elevation and serum gamma-glutamyl transpeptidase in response to adverse job conditions: how are they interrelated? Biol Psychol 1990;31:157 71. 
32. Cheek F, Miller M. The experience of stress for correction officers: a double-bind theory of correctional stress. J Crim Justice 1983;11:105-20.

33. Jacquinet-Salord MC, Lang T, Fouriaud C, Nicoulet I, Bingham A. Sleeping Tablet consumption, self reported quality of sleep, and working conditions. J Epidemiol Community Health 1993;47:64-8.

34. Butat C, Barrit J, Brugère D, Cosset M, Touranchet A, Volkoff
S. Troubles du sommeil en fonction de l'âge et des horaires de travail (enquête ESTEV). Arch Mal Prof Med Trav 1993; 54(3):209-84.

35. Quera-Salva A, Orlic A, Goldenberg F, Guilleminault C. Insomnia and use of hypnotics: study of a French population. Sleep 1991;14(5):386-91.

Received for publication: 29 April 1994 